\title{
Three-Dimensional Facial Model Reconstruction and Plastic Surgery Simulation
}

\author{
Tong-Yee Lee, Yung-Nien Sun, Yung-Ching Lin, Leewen Lin, and Chungnan Lee
}

\begin{abstract}
Facial model reconstruction and surgical simulation are essential to plastic surgery in today's medicine. Both can help surgeons to design appropriate repair plans and procedures prior to actual surgery. In this paper, we exploit a metamorphosis technique in our new design. First, using metamorphosis and vision techniques, we can establish three-dimensional facial models from a given photo. Second, we design several morphing operators, including augmentation, cutting, and lacerating. Experiments show that the proposed algorithms can successfully create acceptable facial models and generate realistically visual effects of surgical simulation.
\end{abstract}

Index Terms - Facial model reconstruction, metamorphosis, morphing operators, surgical simulation.

\section{INTRODUCTION}

I $\mathrm{N}$ HOSPITALS, plastic surgery is a routine procedure to correct congenital deformities or to treat the deformities caused by accidents. Precise presurgical planning can help surgeons to reduce the potential of risks in plastic surgery. In the past, there have been several related researches proposed. Koch et al. [1] proposed algorithms in both presurgical planning and postsurgical evaluation. In this method, the facial surgery is realistically simulated using finite element methods. In [2], physically based modeling was developed to generate facial expression. However, this approach is computationally very expensive; it is not practical in real-time surgical simulation. In forensic medicine, several studies have been proposed for facial reconstruction [3]-[5]. The goal of these studies is to reconstruct the face of a deceased person from the skull of a given cadaver. Additionally, many researchers are interested in developing patient-specific implants [6], [7]. In this paper, we propose algorithms for generating three-dimensional (3D) facial models and for simulating surgical operations. In contrast to previous studies, we exploit the metamorphosis technique to accomplish both tasks. As a result, this new approach generates an acceptable quality of facial models and simulates realism of surgical operations.

Manuscript revised February 23, 1999. This work was supported by the National Science Council, Taiwan, Republic of China, under Grant NSC 88-2213-E-006-012, Grant NSC 88- 2213-E-006-037, Grant NSC 88-2213E-006-011, and Grant NSC 88-2213-E-110-011.

T.-Y. Lee, Y.-N. Sun, and Y.-C. Lin are with the Visual System Laboratory, Department of Computer Science and Information Engineering, National Cheng-Kung University, Tainan, Taiwan, R.O.C.

L. Lin is with Kaohsiung Senior Vocational Commerce School, Kaohsiung, Taiwan, R.O.C.

C. Lee is with the Institute of Computer and Information Engineering, National Sun Yat-Sen University, Kaohsiung, Taiwan, R.O.C.

Publisher Item Identifier S 1089-7771(99)07089-2.
Metamorphosis or warping is a powerful technique to transform one image or a 3-D model into another [8]-[10]. In the past, it has been widely used in the entertainment industry. In [9], two-dimensional (2-D) warping was classified as follows: mesh morphing, field morphing, radial basis functions, thin plate splines, and energy minimization. In this paper, we primarily use a feature-based volume morphing technique [8] in our design. This is a new application of metamorphosis to surgical simulation. This novel approach enables us to establish facial models and simulate surgical operations.

This paper is organized as follows. Section II will briefly review the 3-D metamorphosis technique used in this paper. Section III will present the framework of 3-D facial model reconstruction. The proposed morphing operators and their applications in plastic surgery are described in Section IV. Finally, concluding remarks and trends of future work are given in Section V.

\section{Feature-Based Three-Dimensional Metamorphosis}

The feature-based volume metamorphosis consists of two major steps, warping and blending [8], as illustrated in Fig. 1.

First, it warps the source and target volumes $S$ and $T$ into intermediate volumes according to several user-specified features. These features will define the transition of morphing between $S$ and $T$. The contribution of each feature to morphing is calculated by a weight function. This weight function is in proportion to $d^{-2}$, where $d$ is the distance from the voxel to a corresponding feature. Second, it blends the density of two intermediate volumes to form a morphed volume. For more details, the reader is referred to [8] and [10].

\section{ThreE-Dimensional FACial Model ReCOnStruction}

A good facial model is helpful for plastic surgeons to design repair plans. In some situations, for example, traffic accidents, it is hard to obtain preaccident facial models of patients except for past photos. In this section, we will present a framework of facial model reconstruction. In this framework, the reconstruction can be accomplished from a patient's photo, as shown in Fig. 2. This framework is summarized as follows. We select a generic facial model from a database to fit a given photo. The shape of the generic model is refined to the desired model using the metamorphosis technique and 3-D pose information. We acquire 3-D pose information of the given photo using a computer vision technique [11]. Three major components of 


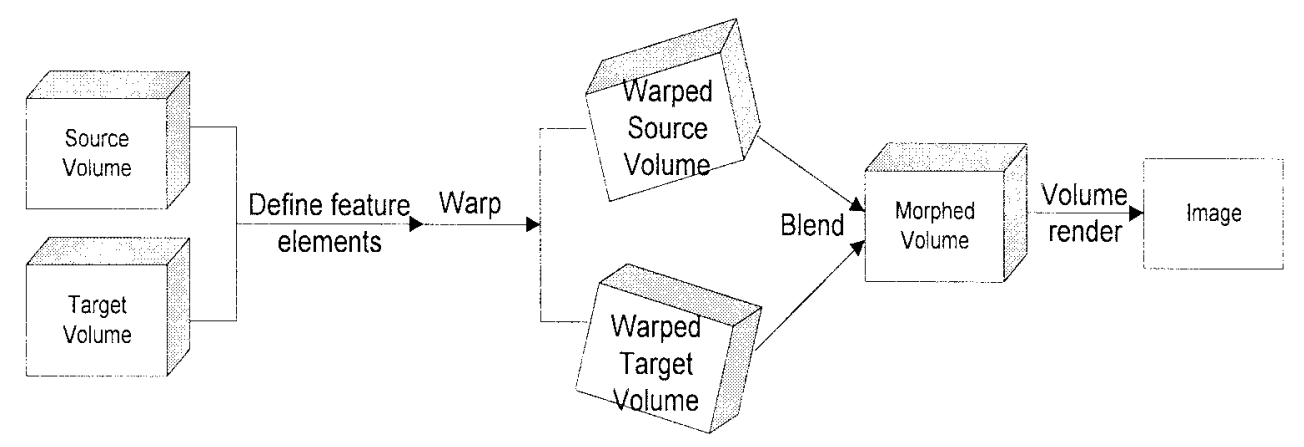

Fig. 1. Feature-based volume metamorphosis.

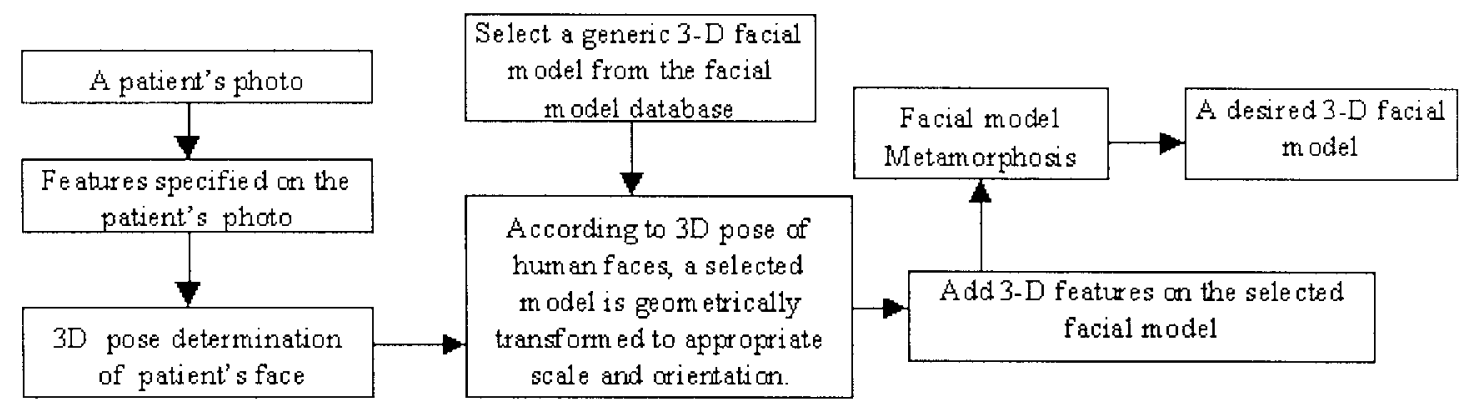

Fig. 2. A framework of 3-D facial model reconstruction.

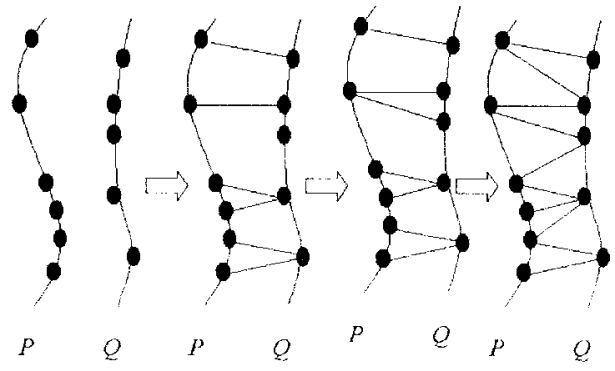

Fig. 3. The triangulation of two consecutive contours, $P$ and $Q$.

this framework will be further explained in the following subsections.

\section{A. Creation of Generic Facial Model}

We create generic facial models from the medical CT head data using the following steps. First, we use image processing packages to extract a set of contours corresponding to the facial surface on CT data. Then, every two consecutive contours will be linked together to approximate the facial model. Assume two consecutive contours $P$ and $Q$ are defined by a sequence of feature points, where $P=\left\{P_{1}, P_{2}, P_{3}, \cdots, P_{m}\right\}, Q=$ $\left\{Q_{1}, Q_{2}, Q_{3}, \cdots, Q_{n}\right\}$ and $m>n$. We achieve their connection using triangular patches in three steps (as shown in Fig. 3). First, we link each point of $P$ to the nearest point on the contour $Q$. Next, each remaining point (not yet linked by $P$ in the first step) of $Q$ will be linked to the nearest point on the contour $P$. After these two steps, we create many patches between $P$ and $Q$. Each patch is either a triangle or a quadrangle. Finally, each quadrangular patch will be divided

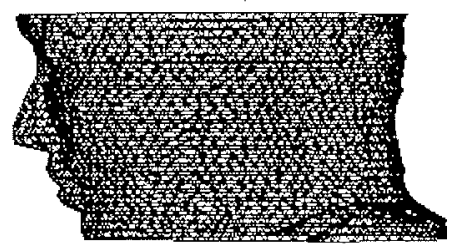

(a)

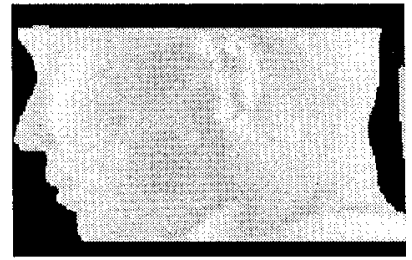

(b)
Fig. 4. CT head representations (a) Wire frame. (b) Volume rendering.

into two triangles as equilateral as possible. The wire frame of a 3-D facial model created using the above method and a volume rendering of the same CT data are shown in Fig. 4. Both representations of the CT head are quite similar.

\section{B. Pose Determination of Human Faces from a Photo}

We acquire pose information of human faces to register a generic facial model with a virtual one (i.e., defined by a photo). For this purpose, we adopt techniques developed in [11] as shown in Fig. 5. In this method, there are four userspecified points on a given photo: $P_{1}, P_{2}$ (two corners of the eyes) and $P_{3}, P_{4}$ (two corners of the mouth), as shown in Fig. 6. Let $P_{5}$ and $P_{6}$ be the centers of $\overline{P_{1} P_{2}}$ and $\overline{P_{3} P_{4}}$. It makes following assumptions: 1) points $P_{1}, P_{2}, P_{3}$ and $P_{4}$ are coplanar; 2) $\overline{P_{1} P_{2}} / \overline{P_{3} P_{4}}$; and 3) $\overline{P_{1} P_{2}} \perp \overline{P_{5} P_{6}}$ and $\overline{P_{3} P_{4}} \perp \overline{P_{5} P_{6}}$. In [11], the required steps to determine 3-D pose information is described as follows. In Fig. 5, both $n_{p}$ and $n_{q}$ are calculated by $\overrightarrow{C p_{1}^{\prime}} \times \overrightarrow{C p_{2}^{\prime}}$ and $\overrightarrow{C p_{3}^{\prime}} \times \overrightarrow{C p_{4}^{\prime}}$, where $\overrightarrow{C p_{1}^{\prime}}=\left(x_{1}^{\prime}, y_{1}^{\prime}, f\right), \overrightarrow{C p_{2}^{\prime}}=\left(x_{2}^{\prime}, y_{2}^{\prime}, f\right), \overrightarrow{C p_{3}^{\prime}}=\left(x_{3}^{\prime}, y_{3}^{\prime}, f\right)$, and $\overrightarrow{C p_{4}^{\prime}}=\left(x_{4}^{\prime}, y_{4}^{\prime}, f\right)$. The parameter $f$ is the focal length of 


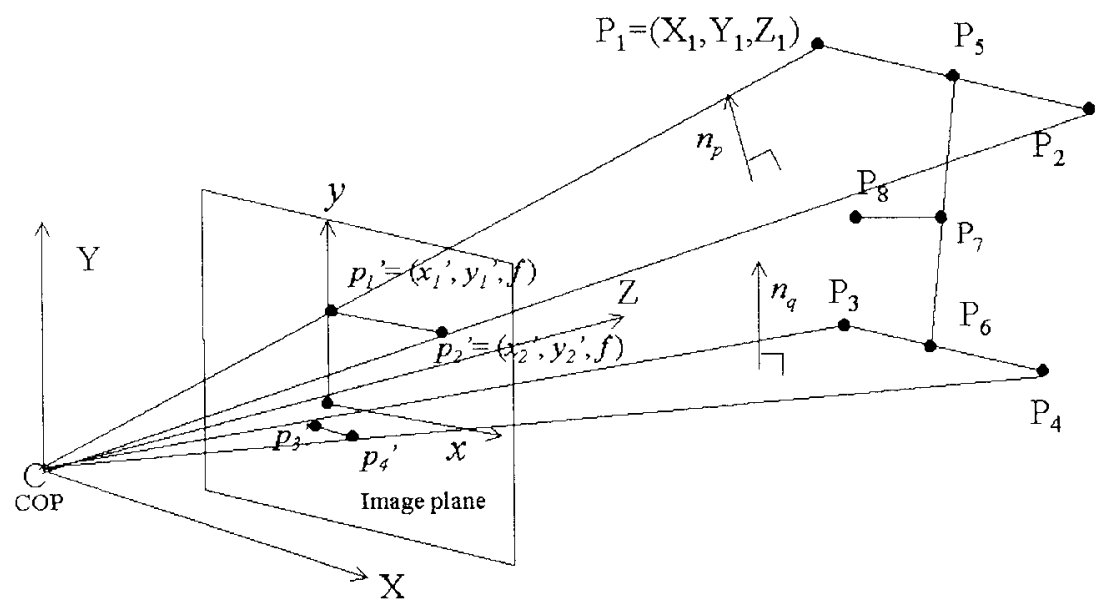

Fig. 5. The perspective projection geometry of a facial model [11].

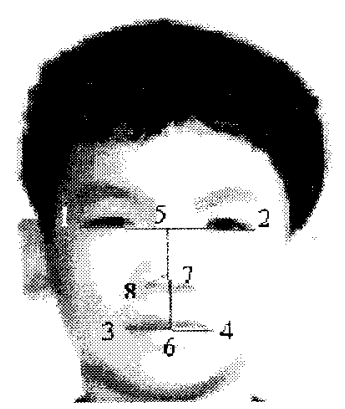

Fig. 6. A photo of a 3-D facial model, where $P_{5}$ and $P_{6}$ are the middle points of line segments $\overline{P_{1} P_{2}}$ and $\overline{P_{3} P_{4}}$, respectively, and $\overrightarrow{P_{7} P_{8}}$ is the same direction as $N_{f}$ (facial normal vector).

the camera. We obtain the eye-line unit vector $N_{e}(u, v, w)$ by $\left(n_{p} \times n_{q}\right) /\left(\left\|n_{p} \times n_{q}\right\|\right)$. Next, we can determine 3-D positions of $P_{1}$ and $P_{2}$ using projective geometry [11] and let $L_{e}=\left\|\overline{P_{1} P_{2}}\right\|$ and $\overrightarrow{P_{1} P_{2}}$ is denoted as $(U, V, W)$. Similarly, since $\overline{P_{1} P_{2}} / / \overline{P_{3} P_{4}}$, we can determine $P_{3}$ and $P_{4}$ by $\overrightarrow{P_{3} P_{4}}$. Notice that $P_{5}$ and $P_{6}$ are the centers of $\overline{P_{1} P_{2}}$ and $\overline{P_{3} P_{4}}$, so their values can be determined also. Now, $P_{3}, P_{4}$, and $P_{6}$ are known and we can determine facial axis unit vector $N_{s}$ by $\overrightarrow{P_{5}} P_{6} /\left|P_{5} P_{6}\right|$. Finally, we can determine the facial normal vector $N_{f}$ by $N_{s} \times N_{e}$. Let $P_{7}$ be the center of $\overline{P_{5} P_{6}}$ and assume the lengths of $\overline{P_{7} P_{6}}$ and $\overline{P_{7} P_{8}}$ are equal. Then, $\overline{P_{7} P_{8}}$ can be derived by $\left\|\overline{P_{7} P_{6}}\right\| \times N_{f}$. The algorithms described in [11] do not need to know $\left\|\overline{P_{1} P_{2}}\right\|$ and $\left\|\overline{P_{3} P_{4}}\right\|$ in advance, and both lengths can be derived from the following relations: $\overline{P_{1} P_{2}} \perp \overline{P_{5} P_{6}}, \overline{P_{3} P_{4}} \perp \overline{P_{5} P_{6}}$, and $\overline{P_{5} P_{6}} \bullet N_{e}=0$. For more details, please refer to [11]. In the following subsection, we will show how to apply these eight points $\left(P_{1}, \cdots, P_{8}\right)$ and three facial vectors $\left(N_{e}, N_{s}, N_{f}\right)$ to perform registration and metamorphosis.

\section{Registration and Metamorphosis of Facial Models}

We need to specify a set of feature pairs to compute metamorphosis. The correct correspondence of each feature pair is the key to the success of facial model reconstruction.

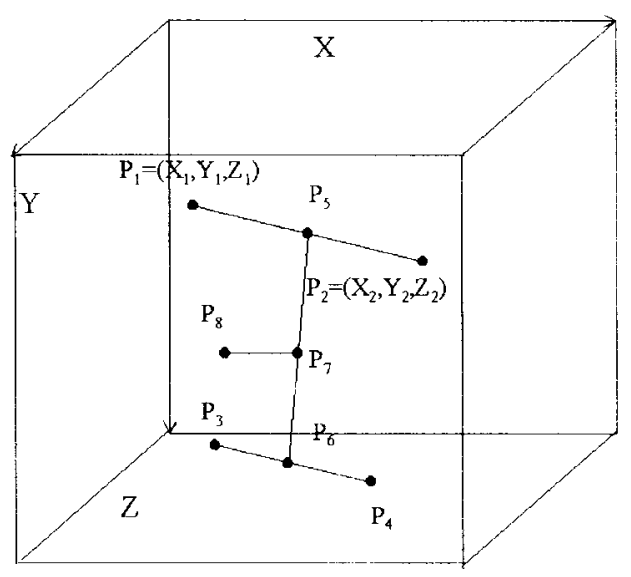

Fig. 7. The generic facial model is transformed to the RCS system.

Therefore, we need to correctly register the generic model with the virtual one (defined by the given photo) prior to metamorphosis. In this paper, we assume that generic and virtual models both have equal lengths of $\overline{P_{1} P_{2}}$. First, we will select eight points, $P_{1} \cdots P_{8}$, on the generic facial model. The reference coordinate system (RCS), as shown in Fig. 7, is defined as follows: the origin is at $P_{7}$, and $N_{e}, N_{s}$, and $N_{f}$ are vectors are the $X, Y$, and $Z$ axes, respectively. Next, we transform both models to the RCS system and thus both models are registered (i.e., they share the same 3-D pose). After this stage, we can safely add additional features to both models to control the transition of the metamorphosis. Notice that this stage must be performed after registration is accomplished. Otherwise, we risk the potential to yield incorrect correspondence of features due to perspective projection. Finally, we calculate metamorphosis using these selected feature pairs.

On a given photo, each feature point $\left(x_{i}, y_{i}\right)$ corresponds to a 3 -D point $\left(t * x_{i}, t * y_{i}, t * f\right)$ on the virtual facial model, where $t$ is a real number and $f$ is the focal length of camera. During registration, it is transformed to the RCS system and it is denoted as $A_{i}$ (i.e., $\left\{X_{i}, Y_{i}, Z_{i}, 1\right\}$ ), 
where

$$
\begin{aligned}
A_{i}= & {\left[\begin{array}{c}
X_{i} \\
Y_{i} \\
Z_{i} \\
1
\end{array}\right]=\left[\begin{array}{cccc}
N_{e x} & N_{e y} & N_{e y} & 0 \\
N_{s x} & N_{s y} & N_{s z} & 0 \\
N_{f x} & N_{f y} & N_{f z} & 0 \\
0 & 0 & 0 & 1
\end{array}\right] } \\
& \cdot\left[\begin{array}{cccc}
1 & 0 & 0 & -X_{7} \\
0 & 1 & 0 & -Y_{7} \\
0 & 0 & 1 & -Z_{7} \\
0 & 0 & 0 & 1
\end{array}\right] \cdot\left[\begin{array}{c}
t_{i} * x_{i} \\
t_{i} * y_{i} \\
t_{i} * f \\
1
\end{array}\right] \\
= & M\left[\begin{array}{c}
t_{i} * x_{i} \\
t_{i} * y_{i} \\
t_{i} * f \\
1
\end{array}\right]
\end{aligned}
$$

and

$$
\begin{aligned}
& \cdot\left[\begin{array}{c}
t_{i} * x_{i} \\
t_{i} * y_{i} \\
t_{i} * f \\
1
\end{array}\right] \\
& =M^{-1} * A_{i} .
\end{aligned}
$$

Next, we determine $t_{i}$ as follows. Assume the correspondence of $A_{i}$ is $C_{i}$ on the generic model. We transform $C_{i}$ by $M^{-1} * C_{i}$. Since we can not recover depth information solely from a single photo, we estimate $Z_{c}$ using $X_{c}$ (i.e., the depths of two models are equal), and then we can have $t_{i}$. In this manner, we can estimate 3-D positions of all feature points, and, thereafter, we can compute metamorphosis.

\section{Results and Discussions}

To verify the proposed framework, Fig. 8 shows an example of facial model reconstruction. The generic 3-D model [Fig. $8(\mathrm{a})]$ is created using algorithms described above, and a single photograph of an individual is shown in Fig. 8(b). First, we select four corners of the eyes and mouth on this photo. Then, both figures were registered using 3-D pose information. Thereafter, additional feature points (marked in both figures) are selected to control the transition of the morphing (13 points in this example). Fig. 8(c) shows the final result in this experiment. Several interesting observations will be pointed out here. The shape of the nose fits very well in this experiment [see Fig. 8(b) and (c)]. The eyes of the CT head are always close, so the generic facial model always lacks detailed geometry of the eyes [see Fig. 8(a) and (c)]. Additionally, there are only four feature points used to control the shape of the eyes. Therefore, it does not yield good results for the eyes. One possible solution to this problem is to exploit multiple views of an individual face rather than a photo to reconstruct facial models. However, generally, it is hard to find several closely related photos of patients, in particular, in accidents. In this situation, a photo on the patient's personal ID is always available. Actually, from our experience, surgeons are satisfied with the current results created from patient ID's. In case of requiring highly realistic model reconstruction,

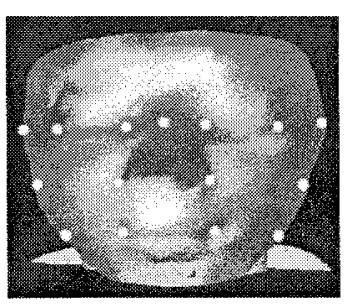

(a)

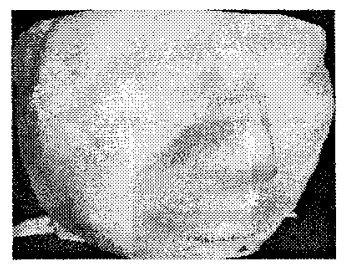

(c)

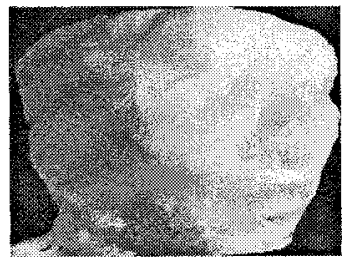

(d)

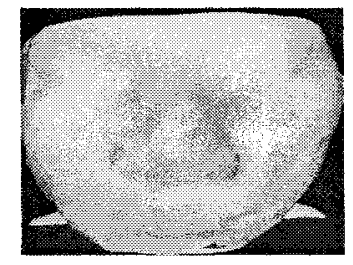

(c)

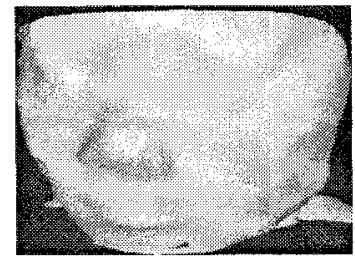

Fig. 8. Model-morphing process. (a) Generic face model (shaded by surface rendering). (b) A given photo marked with feature points. (c) The reconstructed facial model using 13 feature points (side view and front view). (d) The result using 25 feature points (side view and front view).

multiple relevant views of photos and good camera registration are unavoidable. Additionally, the process of extracting the texture maps is necessary for rendering photorealistic images of a reconstructed face model from various viewpoints. These issues are beyond the scope of this paper and will be our future work. Finally, we show one extra result [as shown in Fig. 8(d)] created by adding more features (25 points in this case). We see a better facial model reconstruction is obtained.

\section{Plastic Surgery Simulation Using Morphing}

Three-dimensional metamorphosis has been exploited to create special visual effects. In this paper, we design several morphing operators to simulate surgical operation. These morphing operators globally or locally deform volume data and can the yield desired visual effect. To compute metamorphosis, for a given feature pair $\left(e_{s}, e_{t}\right)$, we will find a point $p^{\prime}$ in the target volume that corresponds to $p$ in the source volume by inverse mapping. We propose three morphing operators, namely, augmentation, cutting, and lacerating, respectively. These manipulations are common in plastic surgery. In our design, the features include points and line segments.

\section{A. Augmentation Operator}

When we want to achieve the visual effect of augmentation on human faces, we can impose an augmentation operator on the patient's facial model. In our design, this operator is 


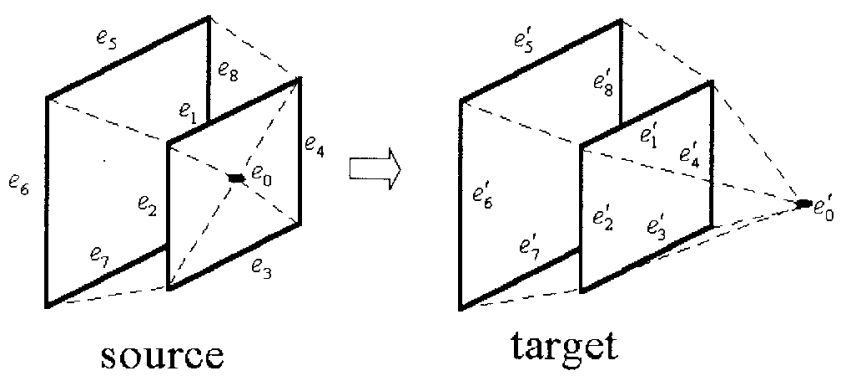

Fig. 9. Augmentation operator.
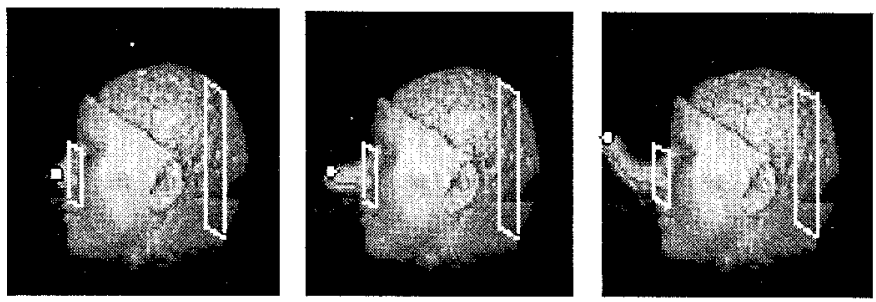

Fig. 10. An example of nose augmentation.

defined by $\left(F_{s}, F_{t}\right)$, where each $F_{i}$ consists of nine features denoted as $\left\langle e_{0}, e_{1}-e_{8}\right\rangle$. The corresponding point $p$ in the source volume can be computed using the following equation:

$$
\frac{\sum_{i=1}^{8} W_{i} p^{\prime}+W_{0} p_{0}}{\sum_{i=0}^{8} W_{i}} .
$$

The design of this operator is shown in Fig. 9. In this design, the $\left(e_{0}, e_{0}^{\prime}\right)$ pair is used to control the effect of augmentation. Note that the morphing effect achieved in [8] is global. Therefore, we need some special arrangement to freeze the area that will not be augmented. The $\left\langle e_{1}-e_{8}\right\rangle$ and $\left\langle e_{1}^{\prime}-e_{8}^{\prime}\right\rangle$ are the same line segments; they are used to freeze the area of volume bounded by these eight line segments. Hence, the positions of $p_{1}-p_{8}$ will be the same as $p^{\prime}$, where $p_{i}$ is the correspondence of $p^{\prime}$ under a feature $e_{i}^{\prime}$. Furthermore, since any $p^{\prime}$ in the area bounded by $\left\langle e_{1}^{\prime}-e_{8}^{\prime}\right\rangle$ is far from $e_{0}^{\prime}$, it is less influenced by this feature point. Therefore, any $p^{\prime}$ contained in this area can be seen as being unchanged (i.e., frozen). Fig. 10 illustrates an example of augmentation. The original MRI head volume data and nine specified features are illustrated in Fig. 10(a). The feature point $e_{0}$ is placed on the nose. Next, in Fig. 10(b), we translate $e_{0}$ to a new position $e_{0}^{\prime}$ that results in an augmentation of the nose. Furthermore, by placing a new feature point, the nose can be further pulled longer [Fig. 10(c)] on the basis of intermediate volume [Fig. 10(b)].

Before we introduce two other operators, we give some background information about them. Our design consists of three steps: 1) for a given $p^{\prime}$ and a feature $e_{i}^{\prime}$, we compute $p_{i}$ as described above; 2) we can further modify $p_{i}$ by $p^{\prime}+$ field $\times\left(p_{i}-p^{\prime}\right)$, if it is required; and 3) we also can modify the weight function, if it is necessary. In the second step, the field is a potential field and its general term is defined by $\left(1-\left(d^{2} / D^{2}\right)\right)^{k}$, where $d$ is the distance from $p^{\prime}$ to a feature $e^{\prime}$. The parameter $D$ is a constant to define the potential, and $k$ is also a constant and its value is dependent on material characteristics.

\section{B. Cutting Operator}

When performing plastic surgery, it is often required to cut away or remove deformities from human faces. This cutting operation can be simulated using morphing as follows. First, we specify two feature line segments $\left\langle L_{s}, L_{s}\right\rangle$ (i.e., the same line segments) on the source volume and $\left\langle L_{1}, L_{2}\right\rangle$ on the target volume, respectively. The angle spanned by $L_{1}, L_{2}$ is used to define the crack on the patient's deformities as shown in Fig. 11(a). In practice, we can just simply give a cutting angle, $\theta$, and then use simple geometry to compute $\left\langle L_{1}, L_{2}\right\rangle$ by means of $L_{s}$. The cutting effect is governed by the following formulas:

$$
\begin{aligned}
p_{i} & = \begin{cases}p^{\prime}, & \text { if } d>D \text { or } u_{i}>1 \\
p^{\prime}+\left(1+\frac{d^{2}}{D^{2}}\right)^{k}\left(p_{i}-p^{\prime}\right), & \text { if } d \leq D\end{cases} \\
u_{i} & =\frac{\left(p^{\prime}-P_{i}\right) \cdot\left(Q_{i}-P_{i}\right)}{\left\|Q_{i}-P_{i}\right\|^{2}} \\
w_{i} & = \begin{cases}0, & \text { if } \overrightarrow{c p^{\prime}} \cdot \overrightarrow{n_{i}}>0 \\
w_{i}, & \text { if } \overrightarrow{c p^{\prime}} \cdot \overrightarrow{n_{i}} \leq 0 .\end{cases}
\end{aligned}
$$

In the above formulas, the correspondence of $p^{\prime}$ will be influenced to a greater extent by a potential field. In contrast to [8], this morphing effect is local rather than global. If $d$ is larger $D, p^{\prime}$ will not be influenced. Additionally, the parameter $u_{i}$ is defined as the position of $p^{\prime}$ along the feature line $\overrightarrow{P_{i} Q_{i}}$. If $u_{i}>1$, it means $p^{\prime}$ is out of the range defined by $\left\langle L_{1}, L_{2}\right\rangle$. Hence, under this condition, $p^{\prime}$ will not be influenced either. This operator generates two cutting planes and their normal vectors are $n_{1}$ and $n_{2}$. Let point $c$ be the center of $L_{1}$ or $L_{2}$. If point $p^{\prime}$ is contained in the cutting area, we assign 0 to the corresponding weight function. In other words, point $p^{\prime}$ is morphed into an empty voxel on the source volume. In our experiment, the parameter $k$ varies from 1 to 4 . Fig. 11(b) shows an example of cutting into an MRI head. In this example, we specify a line segment $L_{s}$ and a cutting angle $\theta$ $\left(60^{\circ}\right)$ to control the cutting effect.

\section{Lacerating Operator}

Another common routine in plastic surgery is the lacerating operation. The surgeons use knives to lacerate a breach on the facial surface. The proposed lacerating operator is composed of two steps. First, we use a cutting operator to generate a breach on facial models. Next, we enlarge this breach by a scaling operator defined by the following equation:

$$
p_{i}= \begin{cases}p^{\prime}, & \text { if } d>D \\ p^{\prime}+\left(1-\frac{d^{2}}{D^{2}}\right)^{2}\left(p_{i}-p^{\prime}\right), & \text { if } d \leq D .\end{cases}
$$

To virtually enlarge a breach, we will specify a line segment pair $\left\langle e, e^{\prime}\right\rangle$ and a potential constant $D$ [as shown in Fig. 12(a)]. 


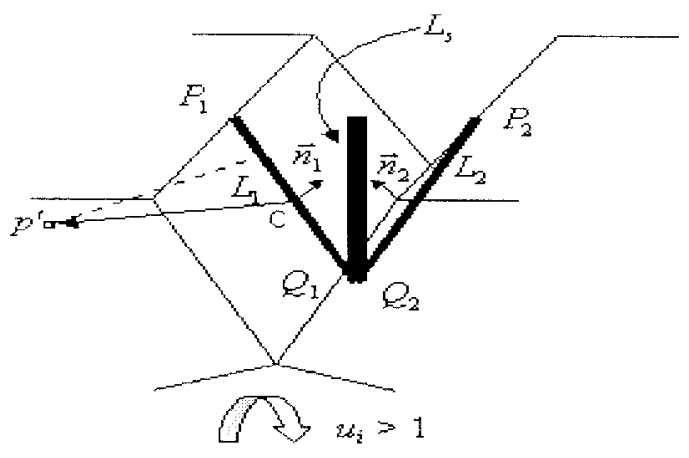

(a)

Fig. 11. (a) Cutting operator. (b) An example of the cutting operation.

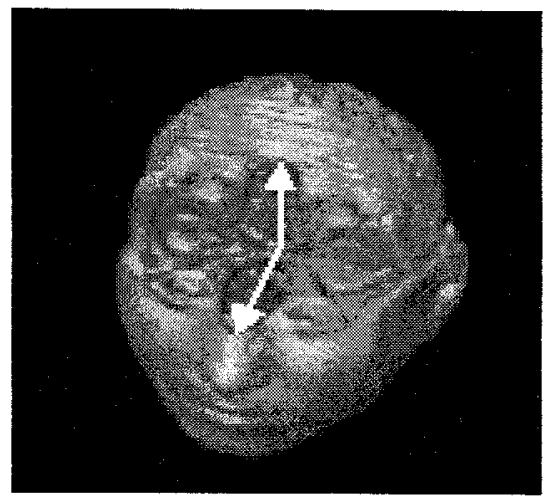

(b)

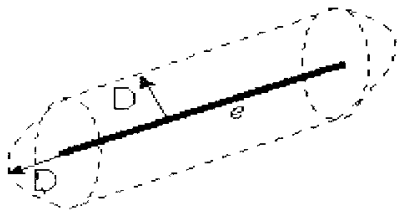

(a)

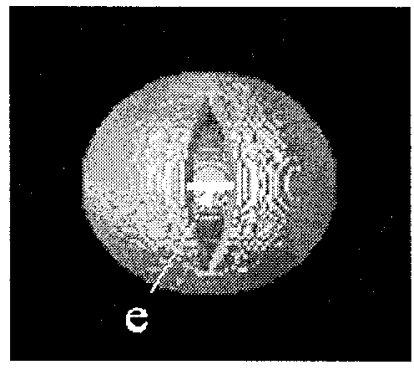

(b)

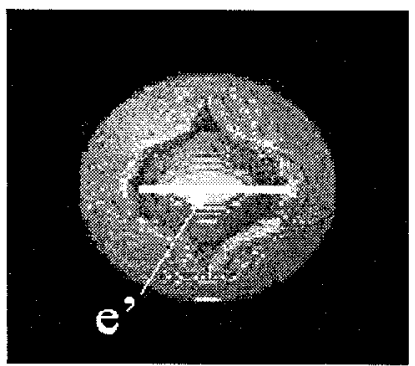

(c)

Fig. 12. (a) A scaling operator. (b), (c) Examples of the lacerating operation.

In this design, actually, the feature $e$ is equal to $e^{\prime}$ but they have different lengths. The distance from $p^{\prime}$ to $e^{\prime}$ is denoted as $d$. If $d$ is less than $D$, then $p^{\prime}$ will be influenced by this operator. In Fig. 12(b) and (c), there are two concentric spheres, where the smaller one is inside the larger one. In this example, we first use a cutting operator to create a crack on the outer sphere and then enlarge the crack using a scaling operator.

\section{CONCLUSION AND FUTURE WORK}

In this paper, we investigate 3-D metamorphosis techniques toward two goals: 1) facial model reconstruction and 2) realistic simulation of surgical operations. To reconstruct a facial model, we first estimate the 3-D pose of a human face from a given photo. Next, using this pose information, we register the generic face model with the given photo. Hereafter, several corresponding features are selected to perform shape refinement on the generic model using the morphing technique. Current results provide acceptable facial models for our collaborating surgeons in their performance of plastic surgery. However, some portion of the face was not successfully reconstructed, for example, the eyes. The main reason is because not enough 3-D information can be recovered from a given photo. Future improvement can be pursued in reconstructing facial models from multiple photos.

We also proposed several morphing operators to simulate surgical operations. These operators can be either global (augmentation) or local (cutting and lacerating). To simulate a surgical operation, we can place suitable operators on damaged parts in an intuitive manner. We can easily use several operators in turn to simulate the desired operation. Finally, we plan to design more types of operators such as twisting. We will use these morphing operators in our surgical simulation project in collaboration with the Hospital of National Cheng-Kung University in Taiwan.

\section{REFERENCES}

[1] R. M. Koch, M. H. Gross, F. R. Carls, D. F. von Buren, G. Fankhauser, and Y. I. H. Parish, "Simulating facial surgery using finite element models," in ACM SIGGRAPH'96 Annu. Conf. Proc., 1996, pp. 421-428.

[2] Y. Lee, D. Terzopoulos, and K. Waters, "Realistic face modeling for animation," in ACM SIGGRAPH'95 Annu. Conf. Proc., 1995, pp. 55-62.

[3] W. A. Aulsebrook, M. Y. Iscan, J. H. Slabbert, and P. Becker, "Superimposition and reconstruction in forensic facial identification-A survey," Forensic Sci. Int., vol. 75, no. 2/3, pp. 101-120, 1995.

[4] S. Miyasaka, M. Yoshino, K. Imaizumi, and S. Seta, "The computeraided facial reconstruction system," Forensic Sci. Int., vol. 74, no. 1/2, pp. $155-165,1995$

[5] D. H. Ubelaker and G. O'Donnell, "Computer assisted facial reconstruction," J. Forensic Sci., vol. 37, pp. 155-162, 1992.

[6] L. Anderson, "Role of rapid prototyping in preoperative planning and patient-specific implant generation," IEEE Eng. Medicine and Biol., pp. 558-559, Jan. 1996.

[7] K. McAloon, Ed., "Rapid prototyping: A unique approach to the diagnosis and planning of medical procedures," SME, MI, 1997.

[8] A. Lerios, C. Garfinkle, and M. Levoy, "Feature-based volume metamorphosis," in ACM SIGGRAPH'95 Annu. Conf. Proc., 1995, pp. 449-456.

[9] S. Lee, G. Wolberg, K.-Y. Chwa, and S. Y. Shin, "Image metamorphosis with scattered feature constraints," IEEE Trans. Visual. Compu. Graphics, vol. 2, pp. 337-354, Dec. 1996.

[10] T.-Y. Lee, Y.-C. Lin, L. Lin, and Y.-N. Sun, "Fast feature-based metamorphosis and operator design," Comput. Graphics Forum, vol. 17, no. 3, pp. C-15-20, 1998.

[11] S.-Y. Ho and H.-L. Hunag, "An analytic solution for the pose determination of human faces from a monocular image," in Proc. 1997 IPPR Conf. on CVGI, Taiwan, 1997, pp. 499-506. 
Tong-Yee Lee, for photograph and biography, see p. 150 of the June 1999 issue of this TRANSACTIONS.

Yung-Nien Sun, for photograph and biography, see p. 150 of the June 1999 issue of this TRANSACTIONS.

Yung-Ching Lin was born in Tainan, Taiwan. He received the B.S. degree from the Department of Computer Science and Information Engineering from Feng Chia University, Taichung, Taiwan, in 1996, and the M.S. degree from the Department of Computer Science and Information Engineering from National Cheng-Kung University, Tainan, Taiwan, in 1998.

His research interests are digital image processing, computer graphics, and object-oriented programming.

Leewen Lin received the B.S. degree in computer education from National Taiwan Normal University in 1991 and the M.S. degree in computer science and information engineering from National Sun Yat-Sen University, Kaohsiung, Taiwan, in 1998.

She is currently a teacher at Kaohsiung Senior Vocational Commerce School, Taiwan, Republic of China. Her research interests include computer graphics and parallel visualization.
Chungnan Lee received the B.S. and M.S. degrees in electrical engineering from National Cheng Kung University, Tainan, Taiwan, in 1980 and 1982, respectively, and the $\mathrm{Ph} . \mathrm{D}$. degree in electrical engineering from the University of Washington, Seattle, in 1992.

$\mathrm{He}$ is an Associate Professor in the Institute of Computer and Information Engineering at National Sun Yat-Sen University, Kaohsiung, Taiwan, since 1992. Prior to joining the faculty, he was a System Manager of Intelligent System Laboratory, a Teaching Assistant, and a Research Associate, while pursuing his graduate studies at the University of Washington. His current research interests include computer vision, character recognition, computer graphics, Web and Java computing, Web-based knowledge discovery, computer-supported collaborative work, and parallel computing. 